\title{
Hepatosplenic lymphoma presenting initially as hemophagocytic syndrome in a 21-year-old man with Crohn's disease: A case report and brief literature review
}

\author{
Justin Côté-Daigneault MD, Edmond-Jean Bernard MD
}

\begin{abstract}
$\mathrm{T}_{\mathrm{n}, \mathrm{m}}$ he present report describes a case involving a 21-year-old man diagnosed with Crohn's disease 10 years previously. A diffuse mild to moderate colitis was found at colonoscopy. Different treatments based on 5-aminosalicylic acid, prednisone, methotrexate and purinethol were attempted but did not induce remission. Five years later, a combination of purinethol $25 \mathrm{mg}$ per day and infliximab $350 \mathrm{mg}$ every six to seven weeks proved to be successful.

Four years later, the patient was admitted to hospital with fever $\left(39^{\circ} \mathrm{C}\right)$ and bicytopenia, without any gastrointestinal symptoms. A complete blood panel revealed the following: white blood cell count (Wbc) $0.9 \times 10^{9} / \mathrm{L}$ (neutrophils $0.2 \times 10^{9} / \mathrm{L}$ ), a hemoglobin level of $156 \mathrm{~g} / \mathrm{L}$ and a platelet count of $105 \times 10^{9} / \mathrm{L}$. The final doses of purinethol and infliximab were given one month before admission. A bone marrow biopsy was performed and showed hemophagocytic syndrome (Figure 1A). Epstein-Barr virus (EBV) serology (viral capsid antigen [VCA] immunoglobulin [Ig] $\mathrm{M}$ and EBV nuclear antigen [EBNA] IgG) was negative and an abdominal computed tomography scan showed mild hepatosplenomegaly. The patient evolved well clinically and the blood count normalized with filgrastim (Neupogen [Amgen Inc, USA]).

One week later, the patient's blood count showed pancytopenia (Wbc $2.1 \times 10^{9} / \mathrm{L}$, neutrophils $0.1 \times 10^{9} / \mathrm{L}$, hemoglobin level of $133 \mathrm{~g} / \mathrm{L}$ and a platelet count of $42 \times 10^{9} / \mathrm{L}$ ) presenting with abdominal pain. A distal endoscopy showed mild proctitis. Due to the pancytopenia, no treatment was started. EBV serology was positive for EBNA IgG and negative for VCA IgM. No antiviral treatment was started. He was discharged, and outpatient chemotherapy with dexamethasone, etoposide and cyclosporin A (hemophagocytic lymphohistiocytosis - 2004 protocol) was initiated.
\end{abstract}

The patient was readmitted two months later due to Escherichia coli sepsis. Chemotherapy was restarted. Septic shock with multiple organ failure ensued and he was transferred to the intensive care unit (ICU). A few months later, when discharged from the ICU, the patient presented with many secondary infections. Another bone marrow biopsy subsequently revealed hepatosplenic lymphoma. EBV was not found on that biopsy. A palliative splenectomy $(25.3 \mathrm{~cm})$ was performed, and the cytology showed the same type of lymphoma with the presence of EBV (Figure 1 B).

After many trials of chemotherapy and multiple infections, the patient died from Klebsiella ozaenae infection.

\section{DISCUSSION}

Over the past few years, immunomodulatory and immunosuppressive agents have become the cornerstone of treatment for severe or refractory Crohn's disease. Antitumour necrosis factor (TNF)-alpha has demonstrated efficacy in inducing and maintaining remission. However, more than $10 \%$ of patients each year discontinue anti-TNF monotherapy either because of side effects or the inefficacy of long-term treatment. Combination therapy may then be considered. Most recently, according to the Study of Biologic and Immunomodulator Naive Patients in Crohn's Disease (SONIC) study group (1), the combination of anti-TNF and azathioprine was associated with greater clinical and mucosal remission compared with either agent individually. Sokol et al (2) also showed a benefit with this combination therapy for effective maintenance of clinical remission. This increased efficacy is partly explained by the reduction of immunogenicity, leading to a decrease in the formation of antibodies against infliximab. In that study, both types of combination
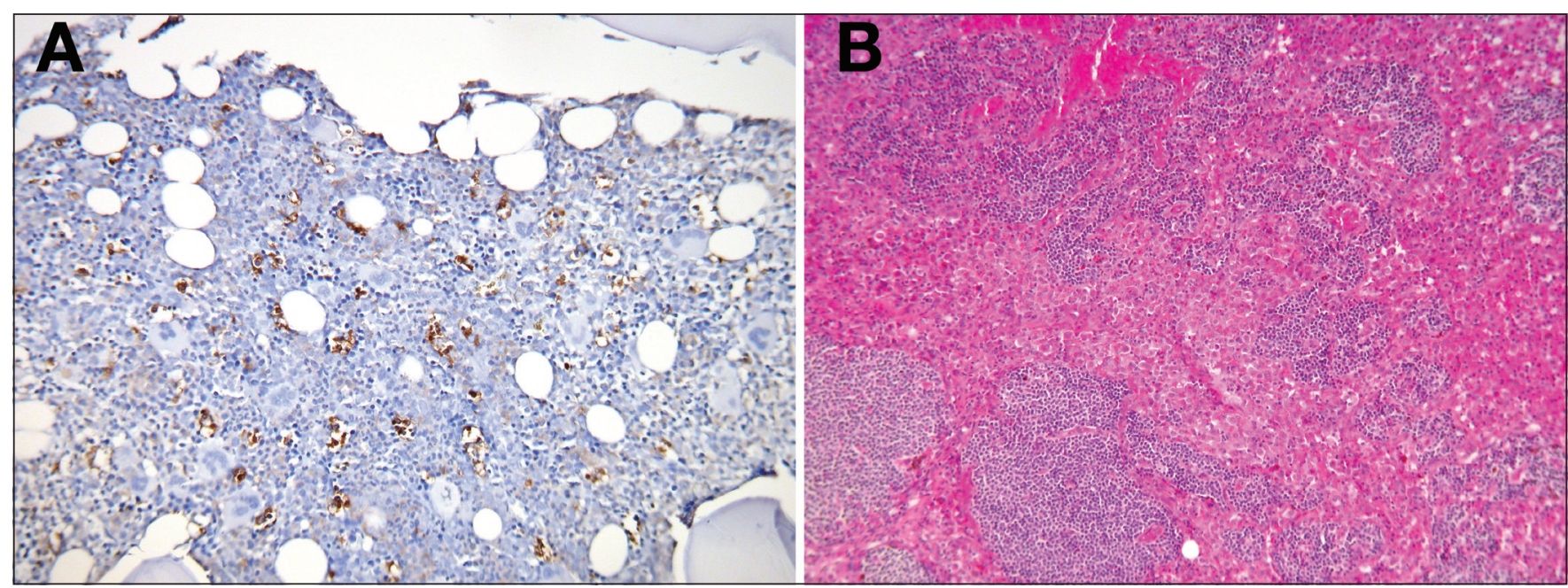

Figure 1) A Bone marrow biopsy showing increased cellularity (90\%), white blood cell hyperplasia and severe hemophagocytosis. A CD68 study revealed histiocyte population infiltration. B Splenic biopsy showing lymphocyte infiltration with irregular nucleus expressing CD2, CD3, CD4 and CD56. Clonal population characterized by $T$ cell receptor-beta and-gamma gene rearrangement, and the presence of Epstein-Barr virus

Gastroenterology Service, Hotel-Dieu (CHUM), University of Montreal, Montreal, Quebec

Correspondence: Dr Justin Côté-Daigneault, Gastroenterology Service, Hotel-Dieu (CHUM), University of Montreal, 3480 St Urbain Street,

Montreal, Quebec H2W 1T8. Telephone 514-890-8000, fax 514-412-7128, e-mail justin.cote-daigneaul@umontreal.ca

Received for publication April 4, 2010. Accepted April 14, 2010 


\section{TABLE 1 \\ Diagnostic criteria for hemophagocytic lymphohistiocytosis}

Major criteria (presence of all five major criteria)

1. Fever

2. Splenomegaly

3. Cytopenia involving two or more lines

4. Hypertriglyceridemia or hypofibrinogemia

5. Hemophagocytosis at biopsy

Minor criteria (criteria A or a combination of B and C can substitute for one major criterion)

A. Low or absent natural killer cell activity

B. Serum ferritin level greater than $500 \mathrm{ug} / \mathrm{L}$

C. Soluble CD25 (sIL-2 receptor) level greater than $2400 \mathrm{U} / \mathrm{L}$

therapies, with methotrexate or azathioprine, provided higher remission rates than monotherapy. However, the combination of infliximab with methotrexate was less effective than its combination with azathioprine.

The long-term safety of this new combination therapy deserves attention because of possible association with several serious side effects including non-Hodgkin's lymphoma. The association between Crohn's disease and lymphoma is tenuous at best. In fact, Crohn's disease alone does not seem to increase the risk of lymphoma; instead, the risk would stem from therapy itself. In rheumatoid arthritis, the incidence of nonHodgkin's lymphoma is not increased with methotrexate (3). In the recently published Cancers Et Sur-risque Associé aux Maladies inflammatoires chroniques intestinales En France (CESAME) registry (4), the incidence of lymphoproliferative disorders was increased in patients with inflammatory bowel disease treated with thiopurines. The association is more difficult to demonstrate for anti-TNF therapy. Indeed, applying a step-up therapeutic strategy, every patient treated with anti-TNF had already been treated with other immunomodulatory agents such as thiopurines. Some

\section{REFERENCES}

1. Colombel JF, Sandborn WJ, Reinisch W, et al. Infliximab, azathioprine, or combination therapy for Crohn's disease. N Engl J Med 2010;362:1383-95.

2. Sokol H, Seksik P, Carrat F, et al. Usefulness of co-treatment with immunomodulators in patients with inflammatory bowel disease treated with scheduled infliximab maintenance therapy. Gut 2010;59:1363-8.

3. Mariette X, Cazals-Hatem D, Warszawki J, Liote F, Balandraud N, Sibilia J. Lymphomas in rheumatoid arthritis patients treated with methotrexate: A 3-year prospective study in France. Blood 2002;99:3909-15.

4. Beaugerie L, Brousse N, Bouvier AM, et al. Lymphoproliferative disorders in patients receiving thiopurines for inflammatory bowel disease: A prospective observational cohort study. Lancet 2009;374:1617-25.

5. Peyrin-Biroulet L, Deltenre P, Suray N de, Branche J, Sandborn WJ, Colombel J-F. Efficacy and safety of tumor necrosis factor studies investigating the risk of lymphoma in the short term have shown no increased risk (5). A combination treatment with thiopurines and antiTNF therapy increased the risk of non-Hodgkin's lymphoma (6). However, investigators found no statistical difference between the incidence rate of lymphoproliferative disorders in the group with combination treatment and the one undergoing thiopurine monotherapy, suggesting that antiTNF monotherapy does not significantly - if at all - increase risk. EBV, a common opportunistic infection in immunocompromised patients, also seems to favour the development of lymphoproliferative disorders.

Hepatosplenic lymphoma belongs to the group of non-Hodgkin lymphomas. Since the first case was described in 1990, approximately 200 have been reported worldwide (7). Including our patient, we found 29 cases in patients with Crohn's disease. All were treated with thiopurines, and the combination therapy with anti-TNF-alpha and thiopurines was found in 23 patients (79\%). Twenty-six patients were men, mostly between 18 and 25 years of age.

Hepatosplenic lymphoma is one of the peripheral $\mathrm{T}$ cell lymphomas, a heterogeneous group constituting $15 \%$ of non-Hodgkin lymphoma (8). It usually presents with hepatosplenomegaly, B symptoms and thrombocytopenia and/or anemia and/or neutropenia. The diagnosis is based on biopsy of affected organs (spleen, liver and bone marrow). The course is rapidly progressive and the prognosis is less than two years of survival. Few data are currently available regarding the treatment.

In this case, hepatosplenic lymphoma was preceded by hemophagocytic syndrome (Table 1) (9). The association between peripheral T cell lymphoma and hemophagocytic syndrome has been described in the literature. However, no case has thus far described hemophagocytic syndrome evolving to hepatosplenic lymphoma in patients with Crohn's disease. EBV infection, hemophagocytic syndrome and B cell lymphoma in patients taking thiopurine for Crohn's disease has already been described (10). Indeed, the present report is the first published case involving hepatosplenic lymphoma, hemophagocytic syndrome and EBV infection in a patient with Crohn's disease undergoing thiopurine and infliximab therapies.

antagonists in Crohn's disease: Meta-analysis of placebo-controlled trials. Clin Gastroenterol Hepatol 2008;6:644-53.

6. Siegel CA, Marden SM, Persing SM, Larson RJ. Risk of lymphoma associated with combination anti-tumor necrosis factor and immunomodulator therapy for the treatment of Crohn's disease: A meta-analysis. Clin Gastroenterol 2010;7:874-81.

7. Ochenrider MG, Patterson DJ, Aboulafia DM. Hepatosplenic T-cell lymphoma in a young man with Crohn's disease: Case report and literature review. Clin Lymphoma Myeloma Leuk 2010;10:144-8.

8. Falchook GS, Vega F, Dang NH, et al. Hepatosplenic gamma-delta T-cell lymphoma: Clinicopathological features and treatment. Ann Oncol 2009;20:1080-5.

9. Egeler RM, Webb D, Winiarski J, Janka G. HLH-2004: Diagnostic and Therapeutic Guidelines for Hemophagocytic Lymphohistiocytosis. ReVision. 2007;(February 2006):124-31.

10. N'guyen Y, Andreoletti L, Patey M, et al. Fatal Epstein-Barr virus primo infection in a 25-year-old man treated with azathioprine for Crohn's disease. J Clin Microbiol 2009;47:1252-4.

The Canadian Journal of Gastroenterology will now consider a limited number of submissions for IMAGE OF THE MONTH. These will be based on endoscopic, histological, radiological and/or patient images, which must be anonymous with no identifying features visible. The patient must consent to publication and the consent must be submitted with the manuscript. All manuscripts should be practical and relevant to clinical practice, and not simply a case report of an esoteric condition. The text should be brief, structured as CASE PRESENTATION and DISCUSSION and not more than 700 words in length. A maximum of three images can be submitted and the number of references should not exceed five. The submission may be edited by our editorial team. 


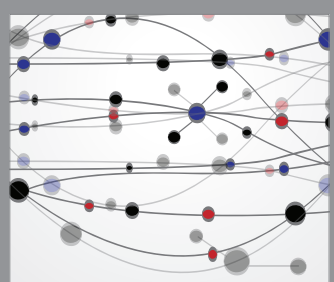

The Scientific World Journal
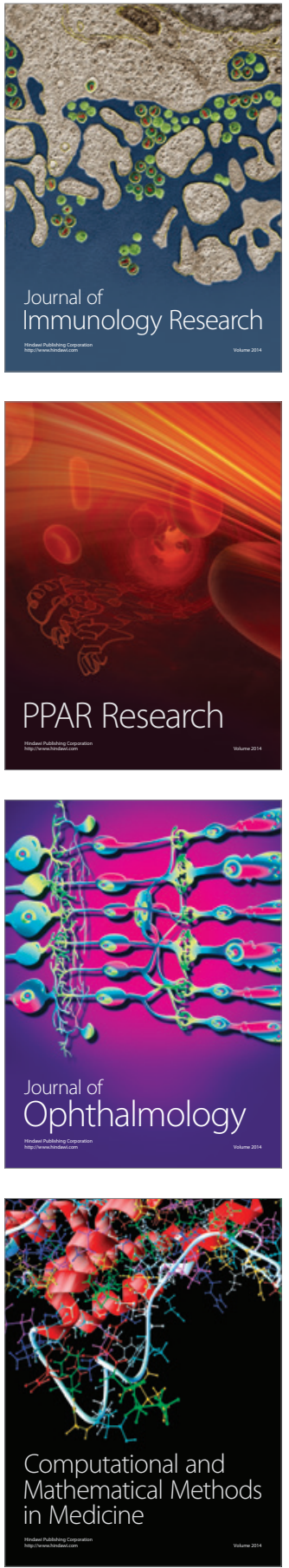

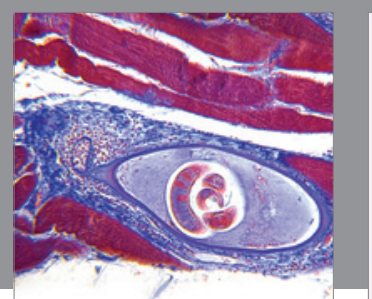

Gastroenterology Research and Practice

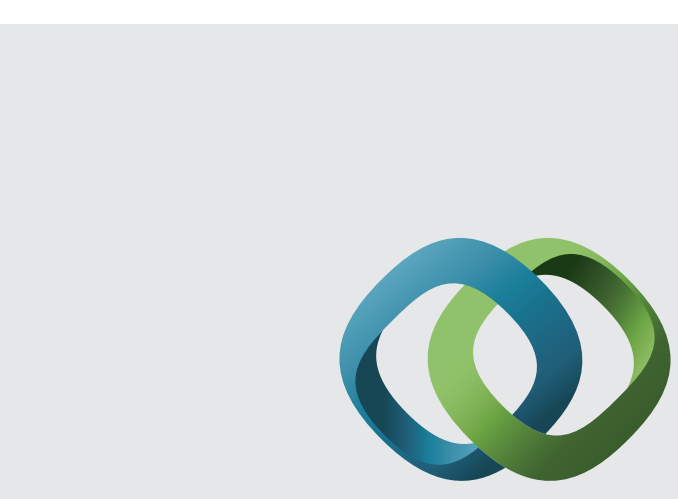

\section{Hindawi}

Submit your manuscripts at

http://www.hindawi.com
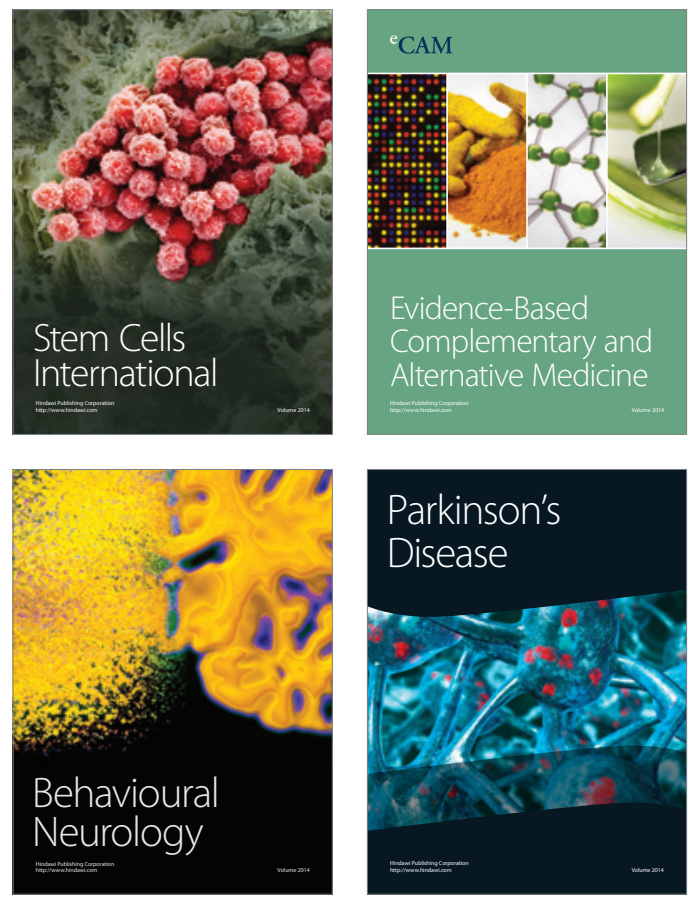
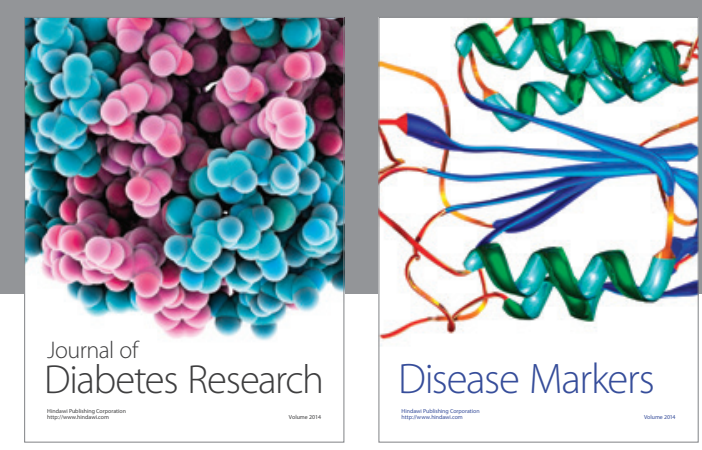

Disease Markers
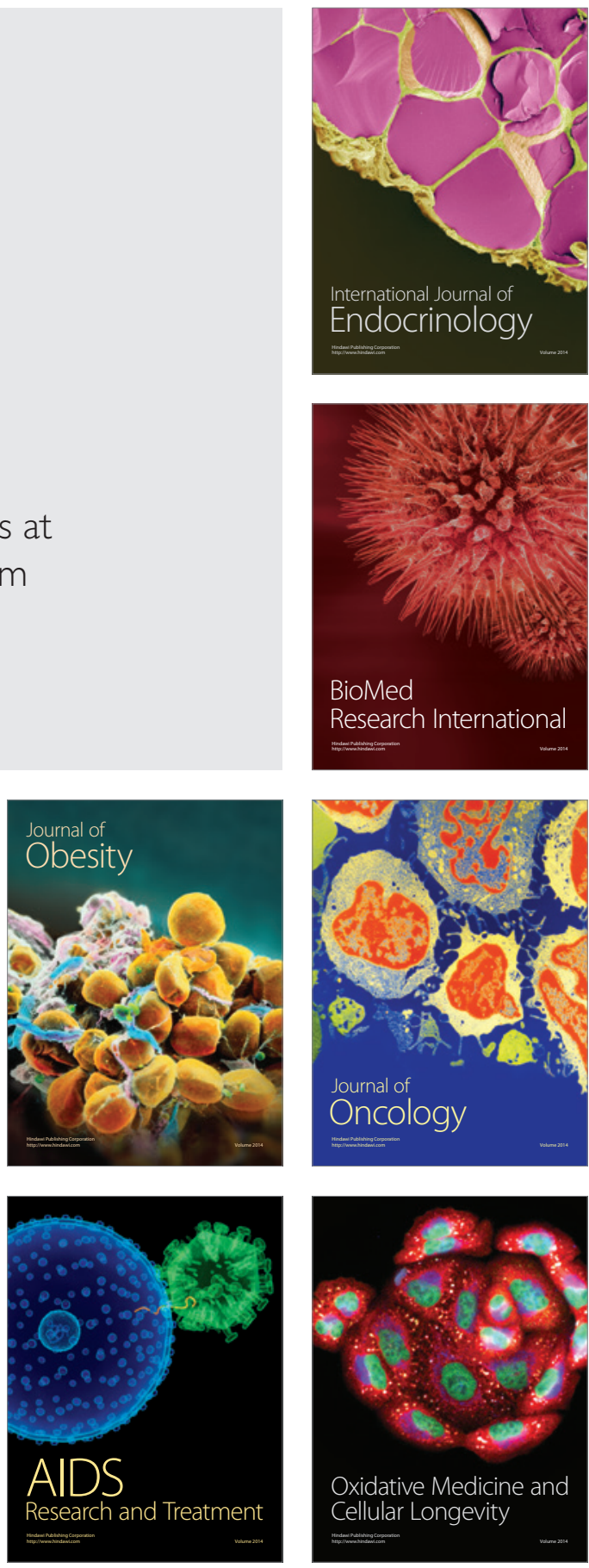